\title{
Switching LPV Control for High Performance Tactical Aircraft
}

\author{
Bei $\mathrm{Lu}^{*}$ and Fen $\mathrm{Wu}^{\dagger}$ \\ North Carolina State University, Raleigh, NC 27695 \\ SungWan Kim ${ }^{\ddagger}$ \\ NASA Langley Research Center, Hampton, VA 23681-2199
}

\begin{abstract}
This paper examines a switching LPV control approach to determine if it is practical to use for flight control designs within a wide angle of attack region. The approach is based on multiple parameter-dependent Lyapunov functions. The full parameter space is partitioned into overlapping subspaces and a family of LPV controllers are designed, each suitable for a specific parameter subspace. The hysteresis switching logic is used to accomplish the transition among different parameter subspaces. The proposed switching LPV control scheme is applied to an F-16 aircraft model with different actuator dynamics in low and high angle of attack regions. The nonlinear simulation results show that the aircraft performs well when switching among different angle of attack regions.
\end{abstract}

\section{Introduction}

I flight control, different performance goals are desirable for different angle of attack regions. For example, in a low angle of attack scenario, pilots desire fast and accurate responses for maneuvering and attitude tracking, whereas in a high angle of attack region, the flight control emphasis lies in the maintainability of aircraft stability with acceptable flying qualities. A modern fighter aircraft usually works in a wide angle of attack region, even near stall or in the post-stall regime. In such a case, it is difficult to design a single robust controller for the entire flight envelope. Typically, the controller is designed in such a way that the performance is compromised in some angle of attack region.

Another issue encountered in flight control is that the actuator dynamics may be different in low and high angle attack regions. It is known that the redundant control effectors, such as thrust vectoring nozzles, are usually incorporated in the high angle of attack region to provide the additional control power. The usual way to generate the thrust vectoring command is a two-step procedure. A controller is designed first based on the generalized control, and the real control inputs are then generated using a control selector. ${ }^{1-4}$ It has not been clearly addressed on how to develop the control law with guaranteed stability and performance by considering the aerodynamic force and thrust force in a unified frame.

To avoid those problems, a method of articulating among multiple controllers according to the evolution of angle of attack was incorporated. The research utilized a switching LPV control technique ${ }^{5}$ to design a family of controllers, each suitable in different angle of attack regions, and switch among them according to the evolution of angle of attack. The whole framework is based on LPV systems because of their relevance to nonlinear systems. The proposed switching LPV control technique is the generalization of results in switched LTI systems. Obviously, stability is an important and challenging problem in switched systems, and it has received considerable attention in recent literature, Refs. 6-8. For a family of stable LTI systems, the existence of a common Lyapunov function provides sufficient conditions for stability of switched systems under arbitrary switching sequences. ${ }^{8,9}$ However, this kind of stability guarantee is deemed too conservative

\footnotetext{
*Graduate Student, Dept. of Mechanical and Aerospace Engineering. AIAA student member.

${ }^{\dagger}$ Corresponding author, Assistant Professor, Dept. of Mechanical and Aerospace Engineering. AIAA Member. E-mail: fwu@eos.ncsu.edu, Phone: (919) 515-5268, Fax: (919) 515-7968.

${ }^{\ddagger}$ Research Engineer, Dynamics and Control Branch. AIAA Associate Fellow.
} 
where a particular switching logic is concerned. For a restricted class of switching signals, multiple Lyapunov functions have proven very useful in stability analysis. Specifically, non traditional stability conditions have been developed using either piecewise continuous Lyapunov functions ${ }^{10-13}$ or discontinuous Lyapunov functions. ${ }^{14,15}$ In the former case, the values of Lyapunov functions corresponding to the active subsystems form a decreasing sequence. This constraint is relaxed in the latter case by requiring each Lyapunov function $V_{p}$ to decrease when the $p^{t h}$ subsystem is active. The results of switched LTI systems have been generalized to the analysis and control of switched LPV systems, ${ }^{16}$ which is further extended in Ref. 17 by introducing average dwell time switch logic. ${ }^{18}$ The stability of switched LPV systems was analyzed by multiple parameterdependent Lyapunov functions, which are allowed to be discontinuous at the switching surfaces. Recently, the authors have developed switching LPV control techniques under hysteresis switching logic and average dwell time switching logic. ${ }^{5}$

In this research, multiple parameter-dependent Lyapunov functions, ${ }^{17}$ which are similar to multiple Lyapunov functions in switched LTI systems, ${ }^{10,11,13,14}$ are used for analyzing the stability of switched LPV systems. We are also interested in controlled performance, which is another major issue and has not been addressed adequately in most current work. In this research, the performance in each angle of attack region is determined, and an upper bound of the performance over the entire angle of attack region is derived. The angle of attack is taken as one of the scheduling parameters, and therefore the switching event is parameterdependent. For LPV systems, it is conceivable that parameter-dependent switching is more practical than state-dependent or time-dependent switching, both of which are usually encountered in switched LTI systems. The full parameter space is partitioned into overlapping subspaces, and hysteresis switching $\operatorname{logic}^{7,19}$ is used to accomplish the transition from one angle of attack region to another.

The notation is standard. $\mathbf{R}$ stands for the set of real numbers and $\mathbf{R}_{+}$for the non-negative real numbers. $\mathbf{R}^{m \times n}$ is the set of real $m \times n$ matrices. The transpose of a real matrix $M$ is denoted by $M^{T}$. $\operatorname{Ker}(M)$ is used to denote the orthogonal complement of $M$. We use $\mathbf{S}^{n \times n}$ to denote the real symmetric $n \times n$ matrices and $\mathbf{S}_{+}^{n \times n}$ to denote positive-definite matrices. If $M \in \mathbf{S}^{n \times n}$, then $M>0(M \geq 0)$ indicates that $M$ is positive-definite (positive semidefinite) and $M<0(M \leq 0)$ denotes a negative-definite (negative semidefinite) matrix. For two sets $A$ and $B$, the set of $\{x: x \in A$, but $x \notin B\}$ is denoted as $A-B$. For $x \in \mathbf{R}^{n}$, its norm is defined as $\|x\|:=\left(x^{T} x\right)^{\frac{1}{2}}$. The space of square integrable functions is denoted by $\mathcal{L}_{2}$, that is, for any $u \in \mathcal{L}_{2},\|u\|_{2}:=\left[\int_{0}^{\infty} u^{T}(t) u(t) d t\right]^{\frac{1}{2}}$ is finite.

\section{Switched LPV Control Synthesis with Hysteresis Switching}

Consider a generalized open-loop LPV system as a function of the parameter $\rho$. It is assumed that $\rho$ is in a compact set $\mathcal{P} \subset \mathbf{R}^{s}$ with its parameter variation rate bounded by $\underline{\nu}_{k} \leq \dot{\rho}_{k} \leq \bar{\nu}_{k}, k=1,2, \ldots, s$. The parameter value is assumed measurable in real-time. For notational purposes, we denote $\mathcal{V}=\left\{\nu: \underline{\nu}_{k} \leq \nu_{k} \leq \bar{\nu}_{k}\right.$, $k=1,2, \ldots, s\}$, where $\mathcal{V}$ is a given convex polytope in $\mathbf{R}^{s}$ that contains the origin. Suppose that the parameter set $\mathcal{P}$ is compact and partitioned into a finite number of closed subsets $\left\{\mathcal{P}_{i}\right\}_{i \in Z_{N}}$ by means of a family of switching surfaces where the index set $Z_{N}=\{1,2, \ldots, N\}$. In each parameter subset, the dynamic behavior of the system is governed by the equation

$$
\left[\begin{array}{c}
\dot{x} \\
e \\
y
\end{array}\right]=\left[\begin{array}{ccc}
A_{i}(\rho) & B_{1, i}(\rho) & B_{2, i}(\rho) \\
C_{1, i}(\rho) & D_{11, i}(\rho) & D_{12, i}(\rho) \\
C_{2, i}(\rho) & D_{21, i}(\rho) & D_{22, i}(\rho)
\end{array}\right]\left[\begin{array}{l}
x \\
d \\
u
\end{array}\right], \quad i \in Z_{N}
$$

where the plant state $x \in \mathbf{R}^{n}$. $e \in \mathbf{R}^{n_{e}}$ is the controlled output, and $d \in \mathbf{R}^{n_{d}}$ is the disturbance input. $u \in \mathbf{R}^{n_{u}}$ is the control input, and $y \in \mathbf{R}^{n_{y}}$ is the measurement for control. All of the state-space data are continuous functions of the parameter $\rho$. Note that each LPV model should have the same number of states, and the reason will be seen in the sequel. It is also assumed that

(A1) $\left(A_{i}(\rho), B_{2, i}(\rho), C_{2, i}(\rho)\right)$ triple is parameter-dependent stabilizable and detectable for all $\rho$.

(A2) The matrix functions $\left[B_{2, i}^{T}(\rho) D_{12, i}^{T}(\rho)\right]$ and $\left[C_{2, i}(\rho) D_{21, i}(\rho)\right]$ have full row ranks for all $\rho \in \mathcal{P}$.

(A3) $D_{11, i}(\rho)=0$ and $D_{22, i}(\rho)=0$.

Given the open-loop LPV system (1), it is sometimes hard to find one LPV controller working for the entire parameter region based on a single Lyapunov function (quadratic or parameter-dependent). This is 
due to different design objectives and actuator dynamics in different parameter regions. Switching LPV control technique permits using most suitable controllers in different parameter subsets. It will also improve performance and enhance design flexibility. In this paper, we are interested in the problem of designing a family of LPV controllers in the form of

$$
\left[\begin{array}{c}
\dot{x}_{k} \\
u
\end{array}\right]=\left[\begin{array}{cc}
A_{k, i}(\rho, \dot{\rho}) & B_{k, i}(\rho) \\
C_{k, i}(\rho) & D_{k, i}(\rho)
\end{array}\right]\left[\begin{array}{c}
x_{k} \\
y
\end{array}\right], \quad i \in Z_{N}
$$

each suitable for a specific parameter subset $\mathcal{P}_{i}$, and $\mathcal{P}=\bigcup \mathcal{P}_{i}$. Dimension of the controller state is $x_{k} \in \mathbf{R}^{n_{k}}$. Each controller stabilizes the open-loop system with the best achievable performance in a specific parameter region, while maintaining the stability of the closed-loop system under the given switching logic.

When hysteresis switching logic is employed, it is assumed that any two adjacent parameter subsets are overlapped, as shown in figure 1(a). Thus there are two switching surfaces between two adjacent parameter subsets. We use $\mathcal{S}_{i j}$ to denote the switching surface specifying the one-directional move from subset $\mathcal{P}_{i}$ to $\mathcal{P}_{j}$.

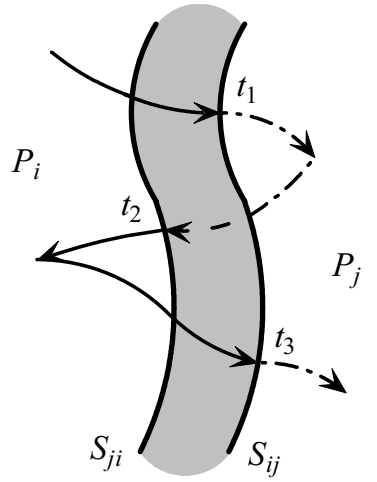

(a) Hysteresis switching region

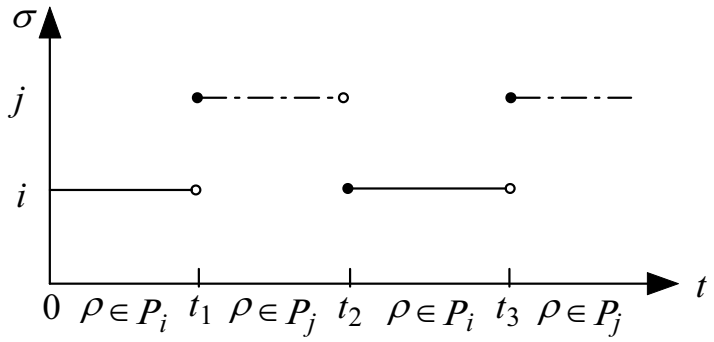

(b) Switching signal

Figure 1. Hysteresis switching region and switching signal $\sigma$.

The switching events occur when the parameter trajectory hits one of the switching surfaces, $\mathcal{S}_{i j}$ or $\mathcal{S}_{j i}$. The evolution of the switching signal $\sigma$ is described as follows: Let $\sigma(0)=i$ if $\rho(0) \in \mathcal{P}_{i}$. For each $t>0$, if $\sigma\left(t^{-}\right)=i$ and $\rho(t) \in \mathcal{P}_{i}$, keep $\sigma(t)=i$. On the other hand, if $\sigma\left(t^{-}\right)=i$ but $\rho(t) \in \mathcal{P}_{j}-\mathcal{P}_{i}$, i.e., hitting the switching surface $\mathcal{S}_{i j}$, let $\sigma(t)=j$. Repeating this procedure, we generate a piecewise constant signal $\sigma$ that is continuous from the right, as shown in figure 1(b). Because $\sigma$ changes its value only after the continuous trajectory has passed through the intersection of adjacent subsets $\mathcal{P}_{i}$ and $\mathcal{P}_{j}$, chattering is avoided. Also, we assume that only a finite number of switches will happen in any finite time interval.

Under switching LPV control, the closed-loop LPV system can be described by

$$
\left[\begin{array}{c}
\dot{x}_{c l} \\
e
\end{array}\right]=\left[\begin{array}{cc}
A_{c l, \sigma}(\rho, \dot{\rho}) & B_{c l, \sigma}(\rho) \\
C_{c l, \sigma}(\rho) & D_{c l, \sigma}(\rho)
\end{array}\right]\left[\begin{array}{c}
x_{c l} \\
d
\end{array}\right]
$$

where $x_{c l} \in \mathbf{R}^{n+n_{k}}$ and

$$
\begin{aligned}
& {\left[\begin{array}{cc}
A_{c l, \sigma}(\rho, \dot{\rho}) & B_{c l, \sigma}(\rho) \\
C_{c l, \sigma}(\rho) & D_{c l, \sigma}(\rho)
\end{array}\right]=\left[\begin{array}{cc|c}
A_{\sigma}(\rho) & 0 & B_{1, \sigma}(\rho) \\
0 & 0 & 0 \\
\hline C_{1, \sigma}(\rho) & 0 & D_{11, \sigma}(\rho)
\end{array}\right]} \\
& +\left[\begin{array}{cc}
0 & B_{2, \sigma}(\rho) \\
I & 0 \\
\hline 0 & D_{12, \sigma}(\rho)
\end{array}\right]\left[\begin{array}{cc}
A_{k, \sigma}(\rho, \dot{\rho}) & B_{k, \sigma}(\rho) \\
C_{k, \sigma}(\rho) & D_{k, \sigma}(\rho)
\end{array}\right]\left[\begin{array}{cc|c}
0 & I & 0 \\
C_{2, \sigma}(\rho) & 0 & D_{21, \sigma}(\rho)
\end{array}\right]
\end{aligned}
$$

A discontinuous Lyapunov function consisting of multiple parameter-dependent Lyapunov functions is used for stability analysis and control design of switched LPV systems. If there exist a family of positivedefinite matrix functions $\left\{X_{i}(\rho)\right\}_{i \in Z_{N}}$, each is smooth over the corresponding parameter subset $\mathcal{P}_{i}$. The 
multiple parameter-dependent Lyapunov functions can then be defined as

$$
V_{\sigma}(x, \rho)=x^{T} X_{\sigma}(\rho) x
$$

where the value of switching signal $\sigma$ represents the active operating region $\mathcal{P}_{i}$ and thus determines the matrix function $X_{i}(\rho)$.

For the closed-loop system (3) under the hysteresis switching logic, if on the switching surface $\mathcal{S}_{i j}$ we have

$$
X_{i}(\rho) \geq X_{j}(\rho)
$$

i.e. the Lyapunov function of the closed-loop system (3) does not increase when switching from $\mathcal{P}_{i}$ to $\mathcal{P}_{j}$. Then we have $V_{i}\left(x_{c l}, \rho\right) \geq V_{j}\left(x_{c l}, \rho\right)$ and the $j$ th controller can be activated safely. Similarly, when switching from $\mathcal{P}_{j}$ back to $\mathcal{P}_{i}$, the condition $X_{j}(\rho) \geq X_{i}(\rho)$ is required on the switching surface $\mathcal{S}_{j i}$ due to the hysteresis effect. Moreover, if the performance is defined as the induced $\mathcal{L}_{2}$ norm of the closed-loop system, the stability and performance of LPV control over each parameter subregion is ensured by ${ }^{20}$

$$
\left[\begin{array}{ccc}
A_{c l, i}^{T}(\rho) X_{i}(\rho)+X_{i}(\rho) A_{c l, i}(\rho)+\sum_{k=1}^{s}\left\{\underline{\nu}_{k}, \bar{\nu}_{k}\right\} \frac{\partial X_{i}}{\partial \rho_{k}} & X_{i}(\rho) B_{c l, i}(\rho) & C_{c l, i}^{T}(\rho) \\
B_{c l, i}^{T}(\rho) X_{i}(\rho) & -\gamma_{i} I_{n_{d}} & D_{c l, i}^{T}(\rho) \\
C_{c l, i}(\rho) & D_{c l, i}(\rho) & -\gamma_{i} I_{n_{e}}
\end{array}\right]<0
$$

where the closed-loop state-space data are described as in (4). The synthesis condition of switched LPV control with hysteresis switching is given in the following theorem.

Theorem 1 Given an open-loop LPV system (1), the parameter set $\mathcal{P}$ and its overlapped partition $\left\{\mathcal{P}_{i}\right\}_{i \in Z_{N}}$, if one of the following equivalent conditions is satisfied:

1. positive-definite matrix functions $R_{i}, S_{i}: \mathbf{R}^{s} \rightarrow \mathbf{S}_{+}^{n \times n}, i \in Z_{N}$ exist such that for any $\rho \in \mathcal{P}_{i}$,

$$
\begin{gathered}
\mathcal{N}_{R, i}^{T}(\rho)\left[\begin{array}{ccc}
R_{i}(\rho) A_{i}^{T}(\rho)+A_{i}(\rho) R_{i}(\rho)-\sum_{k=1}^{s}\left\{\underline{\nu}_{k}, \bar{\nu}_{k}\right\} \frac{\partial R_{i}}{\partial \rho_{k}} & R_{i}(\rho) C_{1, i}^{T}(\rho) & B_{1, i}(\rho) \\
C_{1, i}(\rho) R_{i}(\rho) & -\gamma_{i} I_{n_{e}} & 0 \\
B_{1, i}^{T}(\rho) & 0 & -\gamma_{i} I_{n_{d}}
\end{array}\right] \mathcal{N}_{R, i}(\rho)<0 \\
\mathcal{N}_{S, i}^{T}(\rho)\left[\begin{array}{ccc}
A_{i}^{T}(\rho) S_{i}(\rho)+S_{i}(\rho) A_{i}(\rho)+\sum_{k=1}^{s}\left\{\underline{\nu}_{k}, \bar{\nu}_{k}\right\} \frac{\partial S_{i}}{\partial \rho_{k}} & S_{i}(\rho) B_{1, i}(\rho) & C_{1, i}^{T}(\rho) \\
B_{1, i}^{T}(\rho) S_{i}(\rho) & -\gamma_{i} I_{n_{d}} & 0 \\
C_{1, i}(\rho) & 0 & -\gamma_{i} I_{n_{e}}
\end{array}\right] \mathcal{N}_{S, i}(\rho)<0 \\
\\
{\left[\begin{array}{cc}
R_{i}(\rho) & I_{n} \\
I_{n} & S_{i}(\rho)
\end{array}\right] \geq 0}
\end{gathered}
$$

where $\mathcal{N}_{R, i}(\rho)=\operatorname{Ker}\left[B_{2, i}^{T}(\rho) \quad D_{12, i}^{T}(\rho) \quad 0\right]$ and $\mathcal{N}_{S, i}(\rho)=\operatorname{Ker}\left[\begin{array}{lll}C_{2, i}(\rho) & D_{21, i}(\rho) & 0\end{array}\right]$, and for any $\rho \in \mathcal{S}_{i j}$,

$$
\begin{aligned}
R_{i}(\rho) & \leq R_{j}(\rho) \\
S_{i}(\rho)-R_{i}^{-1}(\rho) & \geq S_{j}(\rho)-R_{j}^{-1}(\rho)
\end{aligned}
$$

2. the inequalities (8)-(10) hold and for any $\rho \in \mathcal{S}_{i j}$,

$$
\begin{aligned}
S_{i}(\rho) & \geq S_{j}(\rho) \\
R_{i}(\rho)-S_{i}^{-1}(\rho) & \leq R_{j}(\rho)-S_{j}^{-1}(\rho)
\end{aligned}
$$

then the closed-loop LPV system (3) is exponentially stabilized by switched LPV controllers in the entire parameter set $\mathcal{P}$, and its performance is maintained as $\|e\|_{2}<\gamma\|d\|_{2}$ with $\gamma=\max \left\{\gamma_{i}\right\}_{i \in Z_{N}}$. 
Proof of the above theorem can be found in Ref. 5. Note that the term $R_{i}^{-1}(\rho)$ appears in condition (12), so the synthesis condition for switching LPV controllers is generally nonconvex. The nonconvex switching LPV synthesis condition is difficult to solve; however, if one enforces the matrix variables $R_{i}(\rho)$ to be continuous on the switching surfaces, then for any $\rho \in \mathcal{S}_{i j}$,

$$
\begin{aligned}
R_{i}(\rho) & =R_{j}(\rho) \\
S_{i}(\rho) & \geq S_{j}(\rho)
\end{aligned}
$$

This implies that the dynamic controller on each switching surface has a same state-feedback gain. The equality constraint (15) can be converted into an LMI condition through a relaxation process

$$
-\epsilon I<R_{i}(\rho)-R_{j}(\rho)<\epsilon I
$$

where $\epsilon$ is a small positive number. Thus the hysteresis switching LPV synthesis conditions become convex and can be solved using efficient LMI optimization algorithms. Similarly, nonconvex condition (13)-(14) can be made convex by enforcing $S_{i}(\rho)=S_{j}(\rho)$ and $R_{i}(\rho) \leq R_{j}(\rho)$ on the switching surface $\mathcal{S}_{i j}$. This corresponds to switching LPV controllers with the same state-estimation gain at switching surfaces.

After solving matrix functions $R_{i}(\rho), S_{i}(\rho)$, the gains of switched LPV controllers can be constructed using the following formula: ${ }^{21}$

$$
\begin{aligned}
A_{k, i}(\rho, \dot{\rho})= & -N_{i}^{-1}(\rho)\left\{A^{T}(\rho)-S_{i}(\rho) \frac{d R_{i}}{d t}-N_{i}(\rho) \frac{d M_{i}^{T}}{d t}\right. \\
& +S_{i}(\rho)\left[A(\rho)+B_{2}(\rho) F_{i}(\rho)+L_{i}(\rho) C_{2}(\rho)\right] R_{i}(\rho)+\frac{1}{\gamma_{i}} S_{i}(\rho)\left[B_{1}(\rho)+L_{i}(\rho) D_{21}(\rho)\right] B_{1}^{T}(\rho) \\
& \left.+\frac{1}{\gamma_{i}} C_{1}^{T}(\rho)\left[C_{1}(\rho)+D_{12}(\rho) F_{i}(\rho)\right] R_{i}(\rho)\right\} M_{i}^{-T}(\rho) \\
B_{k, i}(\rho)= & N_{i}^{-1}(\rho) S_{i}(\rho) L_{i}(\rho) \\
C_{k, i}(\rho)= & F_{i}(\rho) R_{i}(\rho) M_{i}^{-T}(\rho) \\
D_{k, i}(\rho)= & 0
\end{aligned}
$$

where the matrix functions $F_{i}(\rho)$ and $L_{i}(\rho)$ are defined as

$$
\begin{aligned}
& F_{i}(\rho)=-\left(D_{12}^{T}(\rho) D_{12}(\rho)\right)^{-1}\left[\gamma_{i} B_{2}^{T}(\rho) R_{i}^{-1}(\rho)+D_{12}^{T}(\rho) C_{1}(\rho)\right] \\
& L_{i}(\rho)=-\left[\gamma_{i} S_{i}^{-1}(\rho) C_{2}^{T}(\rho)+B_{1}(\rho) D_{21}^{T}(\rho)\right]\left(D_{21}(\rho) D_{21}^{T}(\rho)\right)^{-1}
\end{aligned}
$$

However, to comply with hysteresis switching logic, we need to choose particular realizations of LPV controllers with $M_{i}(\rho)=R_{i}(\rho)$ and $N_{i}(\rho)=R_{i}^{-1}(\rho)-S_{i}(\rho)$ corresponding to the second set of synthesis conditions (or the controller realization with $M_{i}(\rho)=S_{i}^{-1}(\rho)-R_{i}(\rho)$ and $N_{i}(\rho)=S_{i}(\rho)$ corresponding to the third set of synthesis conditions).

\section{Flight Control Example}

The system to be controlled is the longitudinal F-16 aircraft model based on NASA Langley Research Center (LaRC) wind tunnel tests, ${ }^{22}$ which is described by Stevens and Lewis in great detail. ${ }^{23}$ In our research, a simple thrust vectoring model is added in the high angle of attack region to provide additional longitudinal axis control power.

\section{A. Longitudinal Model of F-16 Aircraft with Thrust Vectoring}

The states used to describe motion of the aircraft in longitudinal axis over an entire operating envelope are as follows: $V(\mathrm{ft} / \mathrm{s})$ is the total aircraft velocity, $\alpha(\mathrm{deg})$ is the angle of attack, $q(\mathrm{deg} / \mathrm{s})$ is the pitch rate, and $\theta(\mathrm{deg})$ is the pitch angle. For the original aircraft model, the available control inputs are the throttle setting $\delta_{t h}$ and the elevator angle $\delta_{e}(\mathrm{deg})$. The resulting nonlinear equations of motion in longitudinal axis 
are given as follows:

$$
\begin{aligned}
\dot{V} & =\frac{1}{m}\left(F_{x} \cos \alpha+F_{z} \sin \alpha\right) \\
\dot{\alpha} & =\frac{1}{m V}\left(-F_{x} \sin \alpha+F_{z} \cos \alpha\right)+q \\
\dot{q} & =\frac{M_{y}}{I_{y}} \\
\dot{\theta} & =q
\end{aligned}
$$

where $m$ is the aircraft mass, $F_{x}$ and $F_{z}$ are the force components along $x$ and $z$ body axes respectively, $I_{y}$ is the moment of inertia about the $y$ body axis, and $M_{y}$ is the pitching moment. Note that the throttle setting indirectly affects the states through the power output from the engine. Therefore, the actual power level is also considered as a state variable in longitudinal dynamics, and the detailed dynamical model of the engine can be referred in the NASA data. In addition, $V, q$, and flight path angle $\gamma(=\theta-\alpha)$ are selected as outputs.

The $x$ and $z$ axes forces and pitching moment in Eqs. (22)-(25) contain aerodynamic, gravitational, and thrust components.

$$
\begin{aligned}
F_{x} & =\bar{q} S C_{x, t}-m g \sin \theta+T_{x} \\
F_{z} & =\bar{q} S C_{z, t}+m g \cos \theta+T_{z} \\
M_{y} & =\bar{q} S \bar{c} C_{m, t}+M_{T}
\end{aligned}
$$

where $\bar{q}$ is the dynamic pressure, $S$ is the wing surface area, and $\bar{c}$ is the wing mean aerodynamic chord. A complete description of the total coefficients $C_{x, t}, C_{z, t}$, and $C_{m, t}$ can be found in Ref. 22, which also gives the aerodynamic data in tabular form.

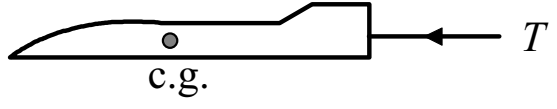

(a) Original aircraft model

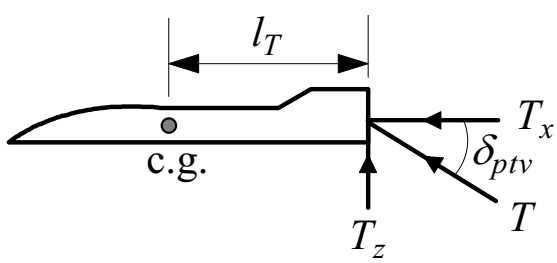

(b) Aircraft model augmented with thrust vectoring

Figure 2. Aircraft model with and without thrust vectoring.

The F-16 is powered by an after-burning turbofan jet engine, which produces a thrust force in the $x$ axis, as shown in figure $2(\mathrm{a})$. In this research, the F-16 aircraft is augmented with a simple thrust vectoring model, which is similar to that in Ref. 2. Denote the thrust vector angle by $\delta_{p t v}$, as shown in figure 2 (b). Then the thrust components along the $x, z$ axes and the pitching moment due to thrust vector are given by

$$
\begin{aligned}
T_{x} & =T \cos \delta_{p t v} \\
T_{z} & =-T \sin \delta_{p t v} \\
M_{T} & =-l_{T} T \sin \delta_{p t v}
\end{aligned}
$$

where $l_{T}$ is the moment arm from the center of gravity to the thrust application point. A more complicated model of thrust vectoring can be found in Refs. 24 and 25, but will not be used in our study.

To develop an LPV representation of the nonlinear F-16 model, we first need to find the wings-level equilibrium points at several flight conditions in the design envelope. The local linear models are then obtained by linearizing the nonlinear equations of motion at those points. The flight envelope of interest covers aircraft speeds between $160 \mathrm{ft} / \mathrm{s}$ and $200 \mathrm{ft} / \mathrm{s}$ and angles of attack between $20^{\circ}$ and $45^{\circ}$. These two variables are used as scheduling parameters in the LPV modeling of F-16 longitudinal dynamics. The points at which the nonlinear model is linearized are marked by a " $\times$ " symbol in figure 3 .

To apply the switching LPV control synthesis technique, the flight envelope is partitioned into two subregions, and the striped area in figure 3 is the overlapped parameter region. In this research, two different 


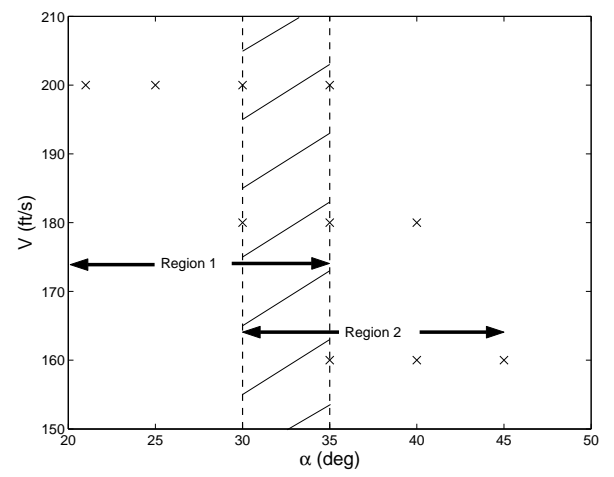

Figure 3. Flight conditions and partitioned flight envelop used for switched LPV model.

sets of actuators are used in the different angle of attack regions. The actuators used in the low angle of attack region $\left(20^{\circ} \leq \alpha \leq 35^{\circ}\right)$ are the throttle and the elevator, and the thrust vectoring nozzle is inactive. The local linear models in this region are based on the original aircraft model, which is corresponding to the case of $\delta_{p t v}=0$. In the high angle of attack region $\left(30^{\circ} \leq \alpha \leq 45^{\circ}\right)$, the thrust vectoring nozzle is incorporated to provide additional force and moment. Therefore, the switching considered in this research is based on the trajectory of the angle of attack, i.e., the controller is switched only when the aircraft flies from one angle of attack region to another.

\section{B. Control Problem Setup}

The design objective is to track the flight path angle command with the tracking error at about $1.25 \%$ of the command in the steady state. The control design is formulated as a model-following problem where the ideal model to be followed is chosen as a second-order filter based on desired flying qualities. A block diagram of the system interconnection for synthesizing the switched LPV controllers is shown in figure 4 , where $P$ is the model set of linearized aircraft dynamics at different trim points and the signal $n$ is a three-dimensional sensor noise.

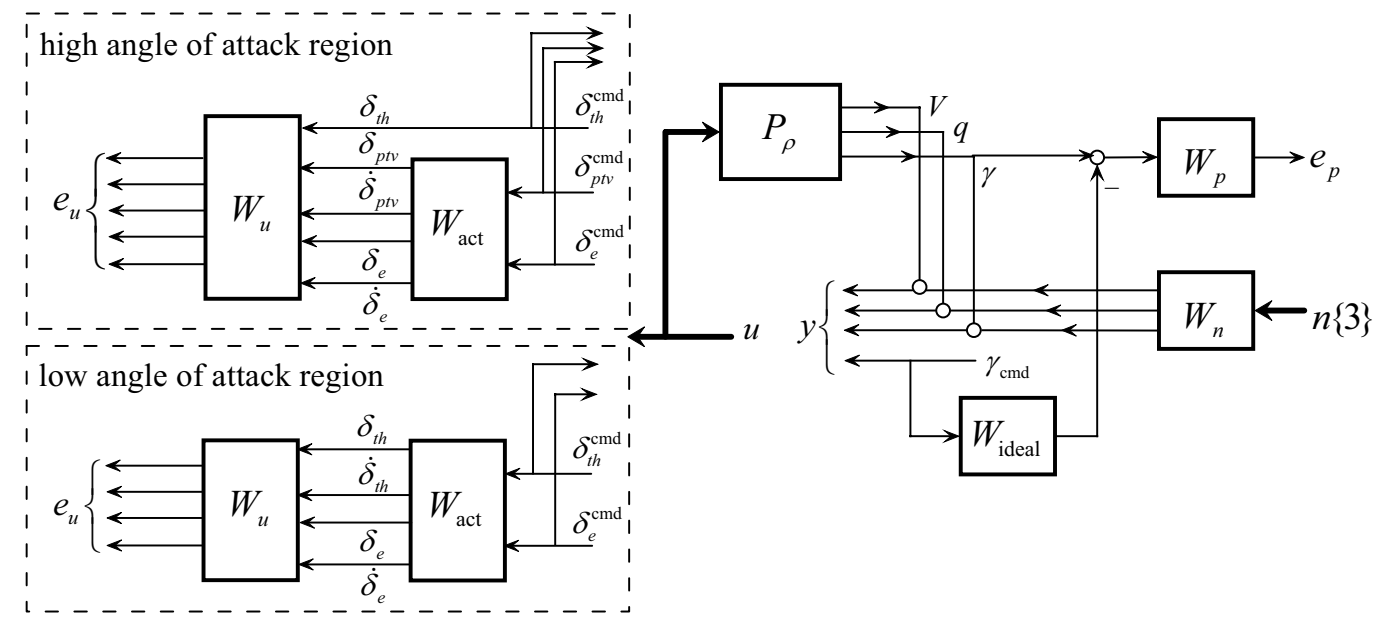

Figure 4. Weighted open-loop interconnection for the F-16 aircraft.

It is noted that the different actuator sets are used in different parameter subspaces. The dynamics of the actuators are modeled as first-order lag filters, and the time constants can be found in Refs. 22 and 2.

$$
\frac{\delta_{t h}}{\delta_{t h}^{\mathrm{cmd}}}=\frac{1}{0.2 s+1}, \quad \frac{\delta_{p t v}}{\delta_{p t v}^{\mathrm{cmd}}}=\frac{1}{0.07 s+1}, \quad \frac{\delta_{e}}{\delta_{e}^{\mathrm{cmd}}}=\frac{1}{0.05 s+1}
$$


In the low angle of attack region, the control inputs are throttle position $\delta_{t h}$ and elevator angle $\delta_{e}$. Both the positions and the rates of control inputs are fed into $W_{u}$ to penalize the control effort. Therefore, the system matrix of $W_{\text {act }}$ is derived as

$$
W_{\text {act }}=\operatorname{diag}\left\{\left[\begin{array}{c|c}
-5 & 5 \\
\hline 1 & 0 \\
-5 & 5
\end{array}\right],\left[\begin{array}{c|c}
-20 & 20 \\
\hline 1 & 0 \\
-20 & 20
\end{array}\right]\right\}
$$

and the weighting function $W_{u}$ is given as

$$
W_{u}=\operatorname{diag}\left\{1,10, \frac{1}{50}, \frac{1}{120}\right\}
$$

In the high angle of attack region, the thrust vector is also activated. In order to use the proposed switching condition, the weighted open-loop LPV plants in the different parameter subspaces must have the same number of the states. Otherwise, the synthesis conditions on the switching surfaces will not hold. Therefore, we ignore the dynamics of the throttle, which has the slowest time constant among the three actuators, and keep the order of $W_{\text {act }}$ as same as that in the low angle of attack region. The related weighting functions $W_{\text {act }}$ and $W_{u}$ are given as follows.

$$
W_{\text {act }}=\operatorname{diag}\left\{\left[\begin{array}{c|c}
-14.28 & 14.28 \\
\hline 1 & 0 \\
-14.28 & 14.28
\end{array}\right],\left[\begin{array}{c|c}
-20 & 20 \\
\hline 1 & 0 \\
-20 & 20
\end{array}\right]\right\}, \quad W_{u}=\operatorname{diag}\left\{1, \frac{1}{35}, \frac{1}{120}, \frac{1}{50}, \frac{1}{120}\right\}
$$

The other common weighting functions are chosen as

$$
W_{p}=\frac{80(s / 5+1)}{s / 0.05+1}, \quad W_{n}=\operatorname{diag}\{0.8,0.6,0.1\}, \quad W_{\text {ideal }}=\frac{0.36}{s^{2}+1.2 s+0.36}
$$

\section{Design Results and Nonlinear Simulations}

Two LPV controllers corresponding to $20^{\circ} \leq \alpha \leq 35^{\circ}$ and $30^{\circ} \leq \alpha \leq 45^{\circ}$ are designed using the switching LPV synthesis condition 3 in Theorem 1. As mentioned before, the synthesis condition (13)-(14) for hysteresis switching control is nonconvex. To avoid numerical complexity in solving the nonconvex problem, we enforce the constraint $S_{i}(\rho)=S_{j}(\rho)$ on the switching surfaces. The resulting synthesis condition then becomes convex and is solvable using efficient LMI optimization algorithm. The multiple parameter-dependent Lyapunov functions at each parameter subset are specified as affine functions of scheduling parameters. That is, we have

$$
R_{i}(\rho)=R_{i}^{0}+\rho_{1} R_{i}^{1}+\rho_{2} R_{i}^{2}, \quad S_{i}(\rho)=S_{i}^{0}+\rho_{1} S_{i}^{1}+\rho_{2} S_{i}^{2}, \quad i=1,2
$$

where $\rho_{1}=\alpha, \rho_{2}=V$, and matrices $R_{i}^{k}$, with $k=0,1,2$ are new optimization variables to be determined. The performance level $\gamma_{i}$ in each parameter subset is 7.94 and 8.03, respectively. Then the $\gamma$ value over the entire parameter set is $\max \left\{\gamma_{1}, \gamma_{2}\right\}$, which represents the "worst-case" LPV control performance using switching LPV control. However, it should be emphasized that the switching LPV synthesis condition is only solved in its relaxed form. It is possible to achieve better performance when nonconvex boundary conditions are included.

For comparison, the performance levels of two other cases are calculated: (1) LPV control in $\left[20^{\circ} 45^{\circ}\right]$; (2) separate LPV control in $\left[\begin{array}{ll}20^{\circ} & 35^{\circ}\end{array}\right]$ and $\left[\begin{array}{ll}30^{\circ} & 45^{\circ}\end{array}\right]$. Both cases are conventional LPV control design problems. In case 1, a single parameter dependent Lyapunov function is used, and the achieved performance level is 8.00, which is almost the same as the switching control. In case 2, the performance levels are 2.88 and 8.00, respectively. The performance levels of case 2 imply that the flight control in the high angle of attack region is much more challenging. For switching LPV control, however, there is no big difference between performance levels of the two subsets. Performance in the low angle of attack region is sacrificed due to convex synthesis conditions. It remains one of the challenging research topics to find an efficient nonconvex algorithm or an alternative synthesis condition.

The switched LPV controllers are also compared with the optimal $\mathcal{H}_{\infty}$ controllers at seven flight conditions, which are used for deriving the LPV model. The $\mathcal{H}_{\infty}$ controllers are synthesized for each flight 
Table 1. Frozen optimal/LPV closed-loop $\mathcal{H}_{\infty}$ norm.

\begin{tabular}{ccc}
\hline \hline Flight condition $[\alpha(\mathrm{deg}), V(\mathrm{ft} / \mathrm{s})]$ & $\mathcal{H}_{\infty}$ control & Switching LPV control \\
\hline$\left[\begin{array}{ll}21, & 200\end{array}\right]$ & 0.8745 & 7.9329 \\
{$\left[\begin{array}{ll}35, & 200\end{array}\right]$} & $0.2685 / 0.2159$ & $7.9453 / 7.9453$ \\
{$\left[\begin{array}{ll}35, & 180\end{array}\right]$} & $0.2282 / 0.1749$ & $7.9488 / 7.9487$ \\
{$\left[\begin{array}{ll}40, & 160\end{array}\right]$} & 0.4492 & 7.9928 \\
{$\left[\begin{array}{ll}45, & 160\end{array}\right]$} & 1.2205 & 8.0287 \\
\hline \hline
\end{tabular}

condition, and their $\mathcal{H}_{\infty}$ norm values are compared with the suboptimal control performance achieved by LPV controllers at specified parameter values. Some of these are provided in table 1. As can be seen from the table, the performance of switching LPV control is sub-optimal for fixed parameters. The comparison results for $0.1^{\circ}$ flight path angle step responses of the fixed parameter $\mathcal{H}_{\infty}$ and switching LPV control are given in figure 5 , where the nonlinear model of the aircraft is used for simulation. The solid lines are the responses using controllers in region 1, the dash-dot lines correspond to the responses using controller in region 2, and the dashed line is the response of the ideal model. Note that in the overlapped parameter region, two LPV controllers are available to use. The data indicate that LPV control provides consistent response characteristics over wide parameter ranges.
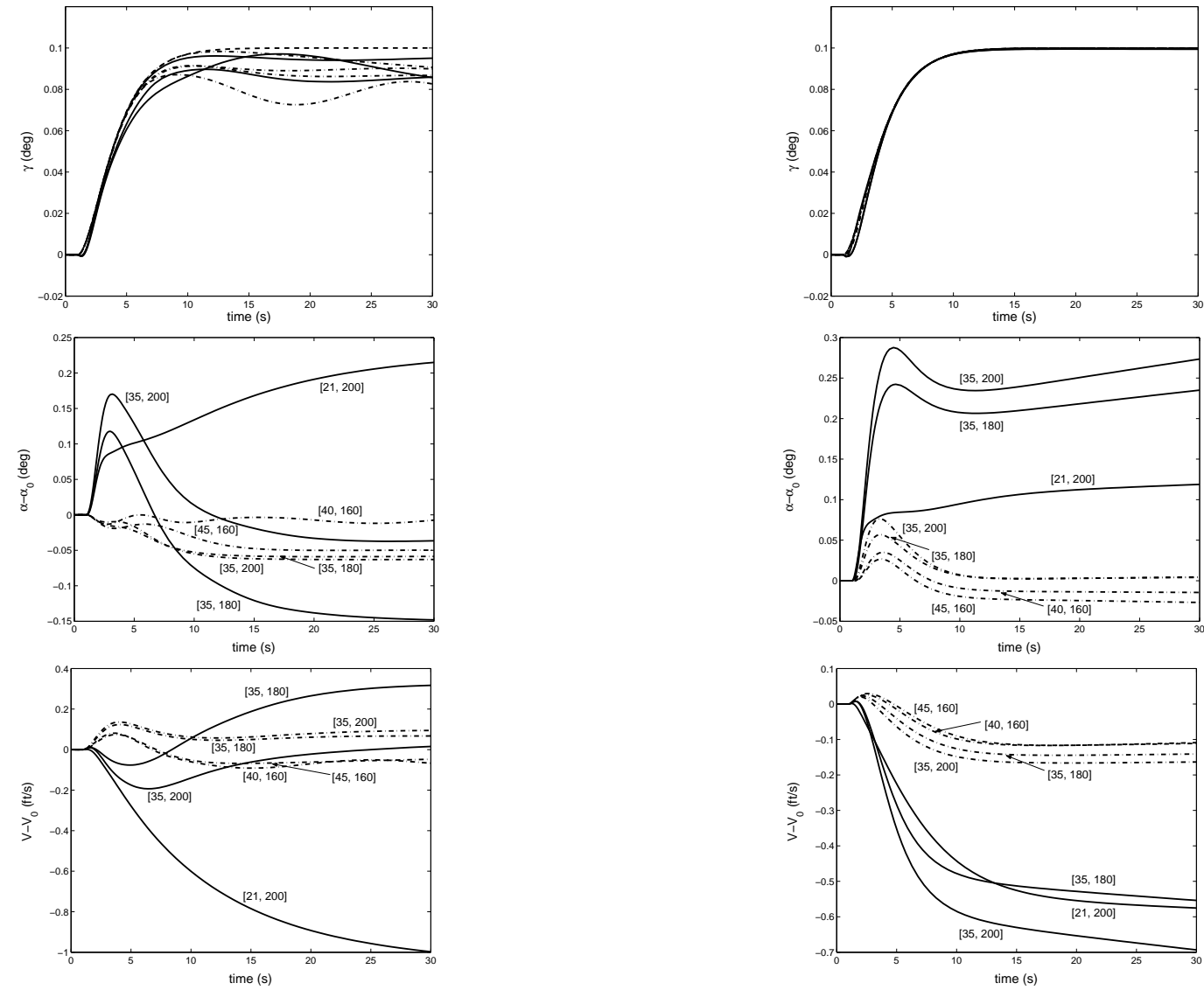

(a) $\mathcal{H}_{\infty}$ control

(b) Hysteresis switching LPV control

Figure 5. Comparison of fixed parameter step response between $\mathcal{H}_{\infty}$ control and hysteresis switching LPV control.

A flight path step input is used to demonstrate performance of the switching control system during the nonlinear simulation, whereas the angle of attack evolves from the low subregion and enters the high 
subregion. The tested flight condition is selected at $V=200 \mathrm{ft} / \mathrm{s}$ and $\alpha=29^{\circ}$. Figure 6 shows the nonlinear responses of the aircraft model to $2^{\circ}$ step input of flight path angle. The dashed line in figure 6 (a) represents the flight path angle response of the ideal model, and the solid line represents achieved system response. Performance over the entire time history is acceptable. Figs. 6(b) and 6(c) show the time history of the scheduling parameters. It can be observed that the switching occurs around $14.25 \mathrm{~s}$, when the angle of attack is about $35^{\circ}$. The responses of actuators are shown in Figs. 6(d)-(f). It is noted that the thrust vector is activated when the aircraft flies over the switching surface $\alpha=35^{\circ}$. During controller switching, there are small glitches for the responses of the throttle and the elevator.

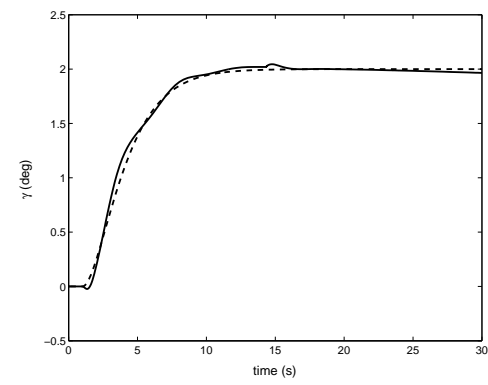

(a) Flight path angle

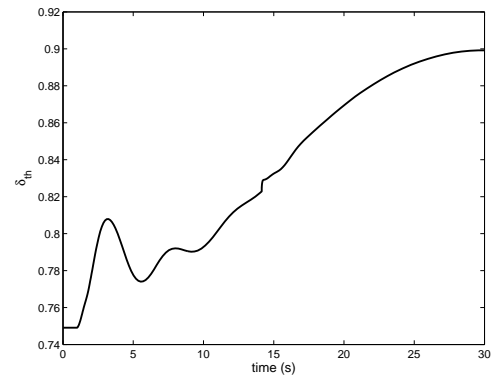

(d) Throttle position

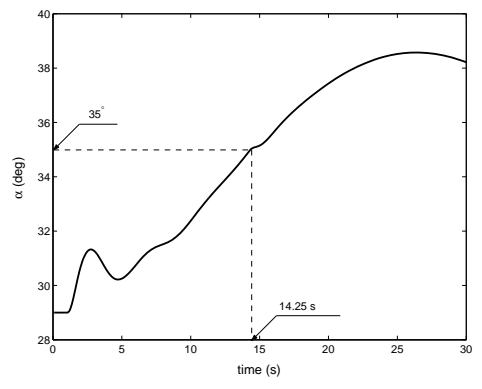

(b) Angle of attack

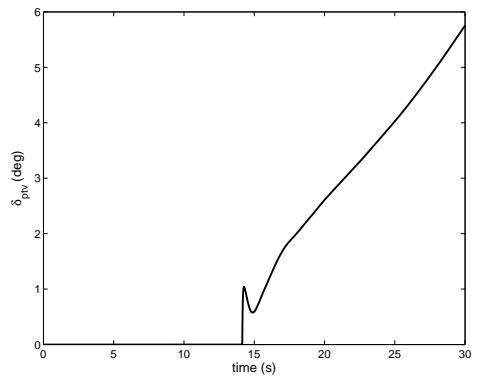

(e) Thrust vectoring nozzle deflection

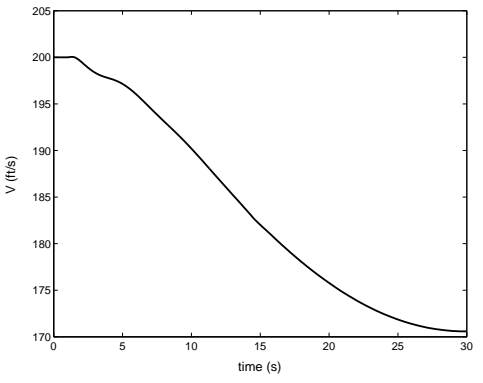

(c) Velocity

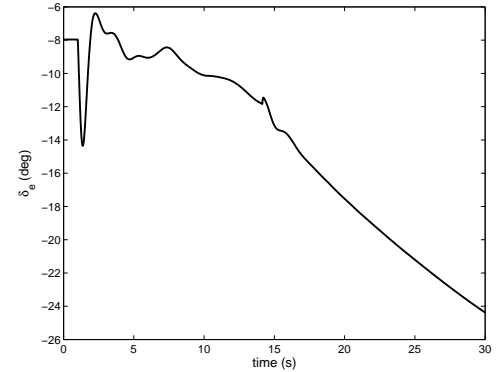

(f) Elevator angle

Figure 6. Nonlinear step response with hysteresis switching.

\section{Conclusion}

A switching LPV control approach based on multiple parameter-dependent Lyapunov functions is presented for flight control design. Hysteresis switching logic is used to avoid the possible transient instability caused by switching among controllers. The proposed technique is used to design a switched control system that achieves the desired flying qualities when switching between low and high angle of attack regions.

The presented flight control design using switched LPV control approach is a good first step toward wide angle of attack maneuvering control design. In this research, the case of different actuator dynamics in different angle of attack regions has been considered. The switching LPV control strategy is capable of providing guaranteed stability and performance for a large flight envelope. For future research, the design objective may also be different in low and high angle of attack regions.

\section{Acknowledgment}

The first two authors would like to acknowledge the financial support for this research by NASA Langley Research Center under grant NAG-1-01119.

\section{References}

${ }^{1}$ Buffington, J.M., Sparks, A.G., and Banda, S.S., "Robust Longitudinal Axis Flight Control for An Aircraft with Thrust vectoring," Automatica, Vol. 30, No. 10, 1994, pp. 1527-1540.

${ }^{2}$ Buffington, J.M., and Enns, D.F., "Flight Control for Mixed-Amplitude Commands," International Journal of Control, 
Vol. 68, No. 6, 1997, pp. 1209-1229.

${ }^{3}$ Reigelsperger, W.C., Hammett K.D., and Banda S.S., "Robust Control Law Design for Lateral-Directional Modes of An F-16/MATV Using mu-Synthesis and Dynamic Inversion," International Journal of Robust and Nonlinear Control, Vol. 7, No. 8, 1997, pp. 777-795.

${ }^{4}$ Reigelsperger, W.C., and Banda, S.S., "Nonlinear Simulation for A Modified F-16 with Full-Envelope Control Laws," Control Engineering Practice, Vol. 6, No. 3, 1998, pp. 309-320.

${ }^{5} \mathrm{Lu}$, B., and Wu, F., "Switching LPV Control Designs Using Multiple Parameter-Dependent Lyapunov Functions," accepted by 2004 American Control Conference, Boston, MA, June 2004.

${ }^{6}$ DeCarlo, R.A., Branicky, M.S., Pettersson, S., and Lennartson, B., "Perspectives and Results on The Stability and Stabilizability of Hybrid Systems," Proceedings of the IEEE, Vol. 88, No. 7, 2000, pp. 1069 - 1082.

${ }^{7}$ Liberzon, D., Switching in Systems and Control, Birkhäuser, Boston, MA, 2003.

${ }^{8}$ Liberzon, D., and Morse, A., "Basic Problems in Stability and Design of Switched Systems," IEEE Control Systems Magazine, Vol. 19, No. 5, 1999, pp. 59-70.

${ }^{9}$ Boyd, S.P., and Yang, Q., "Structured and Simultaneous Lyapunov Functions for System Stability Problems," International Journal of Control, Vol. 50, No. 6, 1989, pp. 2215-2240.

${ }^{10}$ Johansson, M., and Ranzer, A., "Computation of Piecewise Quadratic Lyapunov Functions," IEEE Transactions on Automatatic Control, Vol. 43, No. 4, 1998, pp. 555-559.

${ }^{11}$ Peleties, P., and Decarlo, R., "Asymptotic Stability of m-Switched Systems Using Lyapunov-Like Functions," in 1991 American Control Conference, pp. 1679-1684.

${ }^{12}$ Prajna, S., and Papachristodoulou, A., "Analysis of Switched and Hybrid Systems - Beyond Piecewise Quadratic Methods," in 2003 American Control Conference, Denver, Colorado, June 2003, pp. 2779-2784.

${ }^{13}$ Wicks, M.A., Peleties, P., and DeCarlo, R.A., "Construction of Piecewise Lyapunov Functions for Stabilizing Switched Systems," in Proceedings of 33rd IEEE Conference on Decision and Control, Dec. 1994, pp. 3492-3497.

${ }^{14}$ Branicky, M., "Multiple Lyapunov Functions and Other Analysis Tools for Switched and Hybrid Systems," IEEE Transactions on Automatic Control, Vol. 43, No. 4, 1998, pp. 475-482.

${ }^{15}$ Ye, H., Michel, A.N., and Hou, L., "Stability Theory for Hybrid Dynamical Systems," IEEE Transactions on Automatic Control, Vol. 43, No. 4, 1998, pp. 461-474.

${ }^{16}$ Lim, S., Analysis and Control of Linear Parameter-Varying Systems, Ph.D. Dissertation, Stanford University, 1999.

${ }^{17}$ Lim, S., and Chan, K., "Stability Analysis of Hybrid Linear Parameter-Varying Systems," in 2003 American Control Conference, Denver, Colorado, June 2003, pp. 4822-4827.

${ }^{18}$ Hespanha, J.P., and Morse, A.S., "Stability of Switched Systems with Average Dwell-Time," in Proceedings of 38th IEEE Conference on Decision and Control, Dec. 1999, pp. 2655-2660.

${ }^{19}$ Hespanha, J.P., Liberzon, D., and Morse, A.S., "Hysteresis-Based Switching Algorithm for Supervisory Control of Uncertain Systems," Automatica, Vol. 39, No. 2, 2003, pp. 263-272.

${ }^{20}$ Wu, F., Yang, X.H., Packard, A., and Becker, G., "Induced $\mathcal{L}_{2}$ Norm Control for LPV Systems with Bounded Parameter Variation Rates," International Journal of Robust and Nonlinear Control, Vol. 6, No. 9-10, 1996, pp. 983-998.

${ }^{21}$ Becker, G., "Additional Results on Parameter-Dependent Controllers for LPV Systems," in Proc. 13th IFAC World Congress, 1996, pp. 351-356.

${ }^{22}$ Nguyen, L.T., Ogburn, M.E., Gillert, W.P., Kibler, K.S., Brown, P.W., and Deal, P.L., "Simulator Study of Stall/PostStall Characteristics of A Fighter Airplane with Relaxed Longitudinal Static Stability," NASA Technical Paper $1538,1979$.

${ }^{23}$ Stevens, B.L., and Lewis, F.L., Aircraft Control and Simulation, John Wiley \& Sons, Inc., 1992.

${ }^{24}$ Gal-Or, B., and Baumann, D.D., "Mathematical Phenomenology for Thrust-Vectoring-Induced Agility Comparisons," Journal of Aircraft, Vol. 30, No. 2, 1993, pp. 248-254.

${ }^{25}$ Wise, K.A., and Broy, D.J., "Agile Missile Dynamics and Control," Journal of Guidance, Control, and Dynamics, Vol. 21, No. 3, 1998, pp. 441-449. 